\title{
Innovation for an Inclusive Future
}

\author{
Mark Springett ${ }^{1}$, Mark Rice ${ }^{2}$, Alex Carmichael $^{2}$, and Richard Griffiths ${ }^{3}$ \\ ${ }^{1}$ Interaction Design Centre, Middlesex University Town Hall, \\ Hendon, London, NW4 4BT, UK \\ m.springett@mdx.ac.uk \\ ${ }^{2}$ School of Computing, University of Dundee Dundee, DD1 4HN \\ \{mrice, acarmichael\} @ computing. dundee.ac.uk \\ ${ }^{3}$ School of Computing, Mathematical \& Information Sciences, University of \\ Brighton Watts Building, Moulsecoomb, Brighton, BN2 4GJ, UK \\ r.n.griffiths@brighton.ac.uk
}

\section{Workshop Objectives}

This workshop will focus on setting the agenda for research, practice and policy in support of inclusive design for third generation computer-based products. The next generation of technology represents an unprecedented opportunity to improve the quality of life for groups of users who have previously faced exclusion, such as those with impairments and older citizens. At the same time it risks creating a greater digital divide and further exclusion. How we approach design for this new generation will determine whether or not the third wave will provide positive advances towards an inclusive digital world. We therefore need to put forward both a rationale for inclusive design and provide pointers towards technical development and design practice in support of inclusion. It is our belief that there is not only a strong moral case for design for inclusion but also significant commercial incentive, which may be key to persuading influential players to focus on inclusion. Therefore one of our key objectives is to describe and promote the advantages of designing 'in from the edges' of the user population rather than designing for a notional 'average' user.

The gradual convergence of TV and PC-based services, and the greater interactivity of TV, affords the opportunity to re-think how we can best interact and design services, suitable devices and integration into domestic living spaces and with divergent lifestyles. This convergence and increased capability for service design facilitates creative innovation in which the space of possible applications can be explored. Advances include, but are not limited to, more inclusive provision of entertainment and communication applications associated with interactive TV and Web 2.0. There is a further agenda afforded by the genesis of innovative solutions for independent living, lifelong learning and social inclusion that exploit the potential of TV, mobile, satellite and network technologies.

Yet despite this potential there is an unresolved paradox in the development of digital interactive television and other information appliances within a private, domestic sphere. For example, the replacement of specialized analogue by generalized digital technology has brought about a step change in the flexibility with which broadcast services are consumed. There is consequently an (in principle) improvement in accessibility for disabled audience members. In practice however, there is an increasing 
exclusion of segments of the audience. For example, for people with visual impairments, there is an increased need to read text and interpret graphical displays. For people with motor disabilities there is increased need to make fine manipulations of complex remote controls. For people with cognitive disabilities there is an increased need to make sense of a complex communications technology; one by which they may enter into contractual obligations with a range of programme, service and product providers.

These issues are a reflection in the push towards supporting the youth entertainment market and other markets where early adopters are likely to provide a healthy return from those investing. Consequently, as with previous technology advances there are also groups that are vulnerable to being ignored or left behind as technology and applications advance - despite the potential/promise this new technology can provide. Design has typically targeted the 'ideal user' with the effect that citizens who are dissimilar to that profile are effectively excluded from design thinking. This can be seen as a significant factor in the growing 'digital divide', where the gap between technology adopters and those left behind increases as technology advances.

Design for inclusion potentially rides more easily than ever before with the goals of mainstream end-user technology innovation and development. There is enormous scope for innovation both for enhancement to existing services and exploration of the space of possible new services. However, the conditions and attitudes must be right for this to become a reality. For example, enablement of better inclusion for digital TV services requires development of open and standardised television architectures. This would facilitate the development of a secondary market in accessible technology, perhaps through the adoption of solutions already available in the personal computer market, and perhaps of wholly new solutions specialised for television. This approach has been recognised in the EU FP7 research programme with a strand dedicated to 'Open Systems Reference Architectures, Standards and ICT Platforms for Ageing Well’ (Objective ICT-2009.7.1 ICT \& Ageing, Target Outcome b.)[1].

\section{Reference}

1. European Commission - Information Society and Media: Information and Communication Technologies, Work Programme 2009-2010 (2008) 